\title{
MINOR POSETS OF FUNCTIONS AS QUOTIENTS OF PARTITION LATTICES
}

\author{
ERKKO LEHTONEN AND TAMÁS WALDHAUSER
}

\begin{abstract}
We study the structure of the partially ordered set of minors of an arbitrary function of several variables. We give an abstract characterization of such "minor posets" in terms of colorings of partition lattices, and we also present infinite families of examples as well as some constructions that can be used to build new minor posets.
\end{abstract}

\section{INTRODUCTION}

We investigate the partially ordered set of functions that can be obtained from an arbitrary $n$-variable function $f: A^{n} \rightarrow B$ via identifications of variables. Such functions are called minors of $f$, and they are naturally partially ordered, since some minors of $f$ can be also minors of each other; we shall use the symbol $\downarrow f$ to denote this poset of minors of the function $f$. In fact, the minor relation is a partial order on the set $\mathcal{F}_{A B}$ of all functions of several variables from $A$ to $B$, if we regard functions differing only in inessential variables and/or in the order of their variables as equivalent. Our goal is to characterize the principal ideals $\downarrow f$ of this poset up to isomorphism (see Figure 2). We give the precise definitions in Section 2 here we present only an illustrative example.

Example 1. Let us consider the function $f\left(x_{1}, x_{2}, x_{3}, x_{4}\right)=x_{1} x_{3}+x_{2}+x_{4}$ over the 2-element field. Identifying the first two variables, we obtain the minor $g(x, y, z)=$ $f(x, x, y, z)=x y+x+z$. If we identify the first and the fourth variable, then we get $f(x, y, z, x)=x z+y+x$, which is the same as $g(x, z, y)$, hence we consider this minor to be the same as (or equivalent to) $g$. On the other hand, identifying the first and third variables of $f$, we obtain a new minor $f(x, y, x, z)=x+y+z$, and one can verify that there are no other 3 -variable minors of $f$. Identification of the second and fourth variables yields the minor $h(x, y, z)=f(x, y, z, y)=x z$, which has formally 3 variables, but depends only on 2 of them. Note that $g(x, y, x)=x y$ is equivalent to $h$, hence $h$ is a minor of $g$. Examining all possible variable identifications, we see that $f$ has altogether 6 minors up to equivalence, which form the poset shown in Figure 1 .

Looking only at the Hasse diagram of Figure 1 (ignoring the labels), it is not at all clear, whether there is a function whose minors give this poset, and this is exactly the problem that we consider in this paper. After recalling the necessary definitions and introducing some formalism for minors in Section 2, we present a characterization of such "minor posets" by means of admissible colorings of partition lattices in Section 3. Then, in Section 4 we use this characterization to give some infinite families of examples of minor posets, and we also present some operations that allow us to construct new minor posets from known ones. However, it still remains an open problem to find a finite bounded poset that is not the poset of minors of any function, if there is such a poset at all.

Let us briefly discuss the relevance of minors of functions to universal algebra and multiple-valued logic. Many important properties of an algebraic structure $\mathbb{A}=(A ; F)$ depend only on the clone of term functions of $\mathbb{A}$, not on the set $F$ of basic operations (which is a generating set for this clone). This makes the theory of clones an essential part of universal algebra. Clones of Boolean functions (i.e., functions on the set $\{0,1\}$ )

2010 Mathematics Subject Classification. 06A06 and 05A18 and 08A40. 


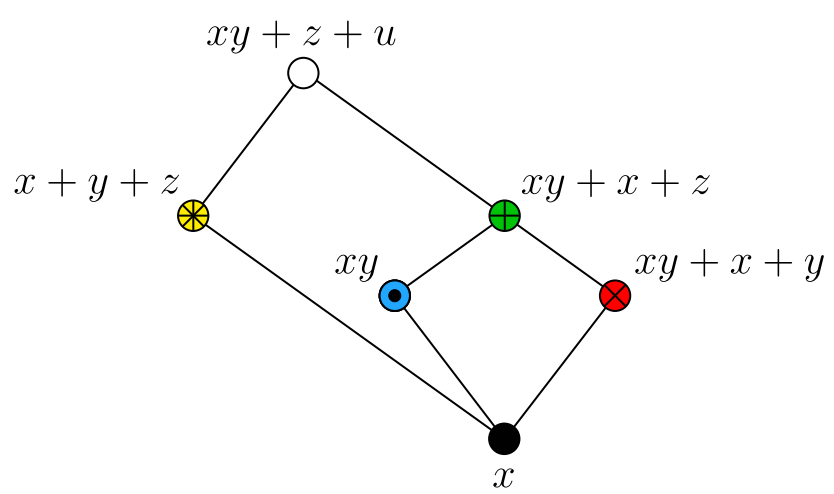

Figure 1. A minor poset.

are obviously relevant for logic [9], and clones of functions on larger sets are central objects of study in multiple-valued logic. Clone theory is essentially the study of compositions of functions of several variables. The simplest kinds of compositions are the ones where we compose a function $f$ with projections. It is easy to see that these are the same as the minors of $f$ (see Example1 and Subsection 2.3). We will see in this paper that even such very simple compositions raise highly nontrivial problems, and we believe that the investigation of these problems contributes to our understanding of clones.

This article is an extended version of the conference paper [7] presented at the 47th IEEE International Symposium on Multiple-Valued Logic, where the main results and sketches of some of the proofs were given.

\section{Preliminaries}

2.1. Posets. For a bounded poset $P$, let $\perp_{P}$ and $\top_{P}$ denote its least and greatest elements; we drop the subscript when there is no danger of ambiguity. The dual of a poset $P$ is the poset $P^{d}$ obtained by reversing the ordering of $P$ (drawing the Hasse diagram of $P$ upside down). The interval $[a, b]$ in $P$ is the set $\{x \in P: a \leq x \leq b\}$. The principal ideal generated by $a \in P$ is the interval $\downarrow a:=\left[\perp_{P}, a\right]$, and the principal filter generated by $a$ is the interval $\left[a, \top_{P}\right]$.

We denote the $n$-element chain by $\mathbf{n}$, the $n$-element antichain by $\overline{\mathbf{n}}$, and $M_{n}$ denotes the bounded poset (in fact, lattice) of size $n+2$ with no comparabilities among its elements except for the top and bottom elements. The ordinal sum (linear sum) of posets $P$ and $Q$ is the poset $P \oplus Q$ obtained by putting $Q$ "on top of" $P$. With this notation we have $\mathbf{n}=\underbrace{\mathbf{1} \oplus \cdots \oplus \mathbf{1}}_{n}$ and $M_{n}=\mathbf{1} \oplus \overline{\mathbf{n}} \oplus \mathbf{1}$.

By a coloring of a poset we mean a surjective map $c: P \rightarrow C$, where $C$ is an arbitrary nonempty set, whose elements are referred to as colors. Given such a coloring, we can introduce a relation $\lambda$ on $C$ by $u \lambda v \Longleftrightarrow \exists a, b \in P: a \leq b$ and $c(a)=u, c(b)=v$. If $\lambda$ is a partial order (which is not always the case), we obtain the "poset of colors" $(C ; \lambda)$, and in this case we will use the symbol $\leq$ instead of $\lambda$. Note that $(C ; \leq)$ can be naturally identified with the poset of equivalence classes with respect to the kernel of the map $c$, hence we shall denote this quotient poset by $P / \operatorname{ker} c$. Let us emphasize that even if $P$ is a lattice (which will always be the case in this paper), the quotient poset $P / \operatorname{ker} c$ is not necessarily a lattice (i.e., $\operatorname{ker} c$ is not always a congruence).

2.2. Set partitions. For any nonempty set $V$, let $\Pi_{V}$ denote the set of all partitions of $V$; if $V=[n]:=\{1, \ldots, n\}$ then we simply write $\Pi_{n}$. Each partition $\alpha \in \Pi_{V}$ corresponds naturally to an equivalence relation $\rho_{\alpha} \subseteq V \times V$. For notational convenience, we will sometimes use the same symbol for a partition and the corresponding equivalence relation, when there is no risk of ambiguity. For example, we denote the block of $\alpha \in \Pi_{V}$ containing $v \in V$ by $v / \alpha$ instead of the more usual notation $v / \rho_{\alpha}$. 
Similarly, we use the symbol ker $h$ not only for the kernel of a map $h: V \rightarrow A$, but also for the corresponding partition in $\Pi_{V}$.

For $\alpha, \beta \in \Pi_{V}$, we say that $\alpha$ is a refinement of $\beta$ and $\beta$ is a coarsening of $\alpha$ (denoted by $\alpha \leq \beta$ ) if every block of $\alpha$ is a subset of some block of $\beta$ (equivalently, $\left.\rho_{\alpha} \subseteq \rho_{\beta}\right)$. The poset $\left(\Pi_{V} ; \leq\right)$ is a lattice, where $\alpha \wedge \beta$ is the partition corresponding to $\rho_{\alpha} \cap \rho_{\beta}$ and $\alpha \vee \beta$ is the partition corresponding to the transitive closure of $\rho_{\alpha} \cup \rho_{\beta}$. The top element of $\Pi_{V}$ is $\top=\{V\}$ and the bottom element is $\perp=\{\{v\}: v \in V\}$. If $\alpha<\beta$ and there is no partition $\xi$ with $\alpha<\xi<\beta$ then $\beta$ is an upper cover of $\alpha(\alpha$ is a lower cover of $\beta$ ), and we shall denote this by $\alpha \prec \beta$. Note that in this case $\beta$ is obtained from $\alpha$ by merging two blocks; in particular, $\vartheta \prec \top$ holds if and only if $\vartheta$ has exactly two blocks.

Ore proved in [8] that every automorphism of $\Pi_{V}$ is induced by a permutation of $V$. It follows immediately that every isomorphism between partition lattices is induced by a bijection between the underlying sets. More precisely, let $V$ and $W$ be nonempty sets, and let $\pi: V \rightarrow W$ be a bijection. For any partition $\alpha=\left\{V_{1}, \ldots, V_{k}\right\} \in \Pi_{V}$, let $\tilde{\pi}(\alpha)=\left\{\pi\left(V_{1}\right), \ldots, \pi\left(V_{k}\right)\right\} \in \Pi_{W}$. Obviously, $\tilde{\pi}: \Pi_{V} \rightarrow \Pi_{W}$ is an isomorphism. With this notation we can recast Ore's theorem in the following form.

Theorem 2 (Ore [8]). For arbitrary sets $V$ and $W$, every isomorphism between $\Pi_{V}$ and $\Pi_{W}$ is of the form $\tilde{\pi}$ for some bijection $\pi: V \rightarrow W$.

Although $\Pi_{V}$ is not a modular lattice if $|V|>3$, the following special case of the isomorphism theorem for perspective intervals in modular lattices does hold (the easy proof is left to the reader).

Fact 3. Let $\alpha, \gamma, \vartheta \in \Pi_{V}$ with $\alpha \leq \vartheta \prec \top$ and $\alpha \prec \gamma \not \leq \vartheta$. If one of the blocks of $\alpha$ is also a block of $\vartheta$, then the following two maps are mutually inverse isomorphisms between the intervals $[\alpha, \vartheta]$ and $[\gamma, \top]$ :

$$
\begin{aligned}
& {[\alpha, \vartheta] \rightarrow[\gamma, \top], \xi \mapsto \xi \vee \gamma} \\
& {[\gamma, \top] \rightarrow[\alpha, \vartheta], \xi \mapsto \xi \wedge \vartheta}
\end{aligned}
$$

Remark 4. The intervals $[\alpha, \vartheta]$ and $[\gamma, \top]$ in Fact 3 are both isomorphic to the partition lattice on $|\alpha|-1=|\gamma|$ elements, hence from Theorem 2 we see that up to permutations of blocks of $\alpha$, the only isomorphism from $[\alpha, \vartheta]$ to $[\gamma, \top]$ is $\xi \mapsto \xi \vee \gamma$.

2.3. Functions and their minors. A function of several variables is a map of the form $f: A^{n} \rightarrow B$, where $A$ and $B$ are arbitrary nonempty sets, and $n$ is a natural number, called the arity of $f$. To avoid degenerate cases, the sets $A$ and $B$ will be assumed to have at least two elements. The set of all such functions (of arbitrary arities) is denoted by $\mathcal{F}_{A B}$. We say that the $i$-th variable of $f$ is essential (or that $f$ depends on its $i$-th variable) if there exist tuples $\mathbf{a}, \mathbf{a}^{\prime} \in A^{n}$ differing only in their $i$-th coordinate such that $f(\mathbf{a}) \neq f\left(\mathbf{a}^{\prime}\right)$.

For $f, g \in \mathcal{F}_{A B}$, we say that $g$ is a minor of $f$ (notation: $g \leq_{\mathrm{m}} f$ ), if there is a map $\sigma:[n] \rightarrow[m]$ such that $g\left(x_{1}, \ldots, x_{m}\right)=f\left(x_{\sigma(1)}, \ldots, x_{\sigma(n)}\right)$, where $n$ and $m$ denote the arities of $f$ and $g$, respectively. It is easy to see that $g \leq_{\mathrm{m}} f$ holds if and only if $g$ can be obtained from $f$ by identification of variables, permutation of variables and/or introduction or deletion of inessential variables. The minor relation is a quasiorder on $\mathcal{F}_{A B}$, and the corresponding equivalence of functions is defined and denoted by $f \equiv g \Longleftrightarrow f \leq_{\mathrm{m}} g$ and $g \leq_{\mathrm{m}} f$. Two functions are equivalent if and only if they can be obtained from each other by permutation of variables and/or introduction or deletion of inessential variables, whereas to form a proper minor $g<_{\mathrm{m}} f$ (meaning $g \leq_{\mathrm{m}} f$ but $\left.g \not \equiv f\right)$, one must identify at least two essential variables. Considering functions only up to equivalence, as we shall do in this paper, one obtains the poset $\left(\mathcal{F}_{A B} / \equiv ; \leq_{\mathrm{m}}\right)$, which is our main object of study. The structure of this poset is quite complicated; for instance, it was shown by Couceiro and Pouzet [5] that it contains a copy of the poset of finite subsets of a countable set (hence a copy of every finite poset) even in the simplest case $A=B=\{0,1\}$ (i.e., in the case of Boolean functions). In 


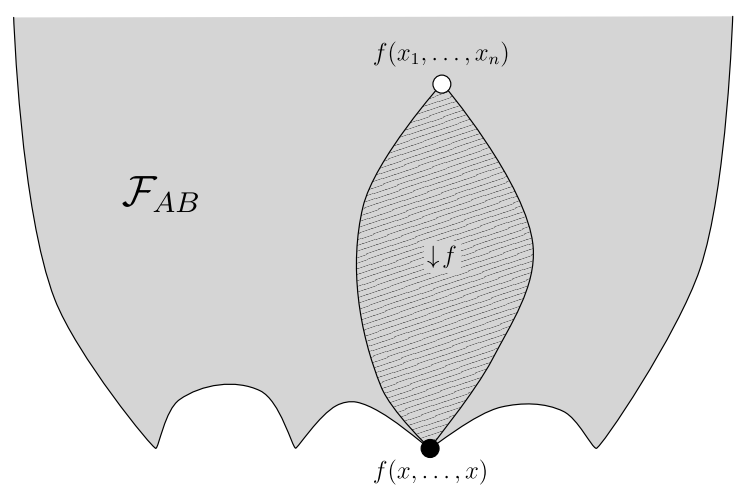

Figure 2. A principal ideal in $\mathcal{F}_{A B}$.

fact, $\left(\mathcal{F}_{A B} / \equiv ; \leq_{\mathrm{m}}\right)$ is universal for the class of countable posets with finite principal ideals, whenever $|B| \geq \min (3,|A|)$ (see Lehtonen, Szendrei [6]).

Here we deal with principal ideals of $\left(\mathcal{F}_{A B} / \equiv ; \leq_{\mathrm{m}}\right)$. The principal ideal $\downarrow f$ generated by a function $f$ consists of the minors of $f$ (up to equivalence), hence we call it the poset of minors of $f$, and we also say that $P$ is a minor poset if there exists a function $f: A^{n} \rightarrow B$ for some sets $A, B$ and for some natural number $n$, such that $P \cong \downarrow f$. Clearly $\downarrow f$ is a finite poset with largest element $f / \equiv$. Although $\mathcal{F}_{A B} / \equiv$ has no least element (but it has several minimal elements), every function $f$ has a least minor, namely the unary function $f(x, \ldots, x)$; see Figure 2, Therefore, every minor poset is a finite bounded poset. We shall denote the class of all minor posets by $\mathcal{M}$, and our main goal is to characterize members of $\mathcal{M}$ by means of a necessary and sufficient condition that does not refer to the existence of a suitable function $f$. In Corollary 18 we establish such a "function-free" characterization; unfortunately, this involves quite an intricate property that is not easy to verify for a concrete poset. Therefore, in spite of this characterization, it is still not clear whether all finite bounded posets are minor posets or not. In Section 4 we present some infinite families of minor posets, and we prove that $\mathcal{M}$ is closed under certain poset constructions.

In order to present the promised characterization, we need to introduce some more abstract formalism for tuples, functions and minors (following Willard [10]). An $n$-ary function from $A$ to $B$ can be viewed as a map $f: A^{V} \rightarrow B$, where $V$ is an arbitrary $n$ element set (whose elements are considered to be the variables of $f$ ), and the elements of $A^{V}$ are maps of the form a: $V \rightarrow A$ (evaluations of variables). Note that in the special case $V=[n]$, the elements of $A^{V}$ can be naturally identified with $n$-tuples, and in this case we get back the usual notion of a function of several variables. We will formulate our results in this usual setting, but in the proofs we will also need the more abstract view of functions allowing arbitrary finite sets as the set of variables.

For $\mathbf{a} \in A^{W}$ and $\sigma: V \rightarrow W$, we can define the composition $\mathbf{a} \circ \sigma \in A^{V}$ by $(\mathbf{a} \circ \sigma)(v)=\mathbf{a}(\sigma(v))$. Minors of $f$ are functions $g: A^{W} \rightarrow B$ that can be given in the form $g(\mathbf{a})=f(\mathbf{a} \circ \sigma)$ for some map $\sigma: V \rightarrow W$. If $\alpha \in \Pi_{V}$ is a partition, then let nat ${ }_{\alpha}$ denote the natural surjection nat $\alpha: V \rightarrow \alpha, v \mapsto v / \alpha$. The map nat induces $_{\alpha}$ a minor $f_{\alpha}: A^{\alpha} \rightarrow B$, which is given by $f_{\alpha}(\mathbf{a})=f\left(\mathbf{a} \circ\right.$ nat $\left.{ }_{\alpha}\right)$ for all $\mathbf{a} \in A^{\alpha}$. Observe that $f_{\alpha}$ is obtained from $f$ by identifying variables belonging to the same block of $\alpha$. Conversely, for every map $\sigma: V \rightarrow W$, the minor $g(\mathbf{a})=f(\mathbf{a} \circ \sigma)$ is equivalent to $f_{\alpha}$ with $\alpha=\operatorname{ker} \sigma$. This shows that it suffices to work with minors of the form $f_{\alpha}$, and we shall record this fact here for reference.

Fact 5. If $f: A^{V} \rightarrow B$ and $g: A^{W} \rightarrow B$ are arbitrary functions, then

$$
g \leq_{\mathrm{m}} f \Longleftrightarrow \exists \alpha \in \Pi_{V}: g \equiv f_{\alpha}
$$




\section{Admissible colorings}

According to Fact 5, every minor of an $n$-variable function $f$ is equivalent to a function $f_{\alpha}$ for some $\alpha \in \Pi_{n}$. This means that we can encode all information about minors of $f$ into a "coloring" $c$ of the partition lattice $\Pi_{n}$, where the color of a partition $\alpha$ is $c(\alpha)=f_{\alpha} / \equiv$. Actually, the only relevant property of this coloring is that two minors receive the same color if and only if they are equivalent. Clearly, we have $\beta \geq \alpha \Longrightarrow f_{\beta} \leq_{\mathrm{m}} f_{\alpha}$. The following easy observation formulates a kind of converse of this statement, showing that we can recover the poset $\downarrow f$ as the quotient of $\Pi_{n}$ by the kernel of the aforementioned coloring $c$.

Proposition 6. For every function $f: A^{n} \rightarrow B$ and for all $\alpha, \beta \in \Pi_{n}$, the function $f_{\beta}$ is a minor of $f_{\alpha}$ if and only if there exists a partition $\gamma \geq \alpha$ such that $f_{\gamma} \equiv f_{\beta}$.

Proof. The "if" part of the statement is obvious. For the "only if" part, assume that $f_{\beta} \leq_{\mathrm{m}} f_{\alpha}$. By Fact 5 this means that there exists a partition $\delta \in \Pi_{\alpha}$ such that $f_{\beta} \equiv\left(f_{\alpha}\right)_{\delta}$. Let $\gamma \in \Pi_{n}$ be the partition obtained by merging the blocks of $\alpha$ that belong to the same block of $\delta$. (More precisely, two elements $u, v \in[n]$ are $\rho_{\gamma}$-related if and only if the $\alpha$-blocks $u / \alpha$ and $v / \alpha$ are $\rho_{\delta}$-related.) Clearly, $\gamma \geq \alpha$ and $\left(f_{\alpha}\right)_{\delta} \equiv f_{\gamma}$, hence $f_{\beta} \equiv f_{\gamma}$.

Corollary 7. For every function $f: A^{n} \rightarrow B$, the poset of minors of $f$ is dually isomorphic to $\Pi_{n} /$ ker $c$ for the natural coloring $c: \Pi_{n} \rightarrow \downarrow f, \alpha \mapsto f_{\alpha} / \equiv$.

Example 8. Let us consider the function $f\left(x_{1}, x_{2}, x_{3}, x_{4}\right)=x_{1} x_{3}+x_{2}+x_{4}$ of Example 1 once more. We have computed there that $f_{12 / 3 / 4} \equiv f_{14 / 2 / 3} \equiv g>_{\mathrm{m}} h \equiv f_{1 / 24 / 3}$ and $f_{13 / 2 / 4}$ is incomparable to $g$ and $h$. (Here we use a simplified, but hopefully clear notation for partitions.) Calculating $f_{\alpha}$ for all the 15 partitions of $[4]=\{1,2,3,4\}$, we get a coloring of $\Pi_{4}$ with 6 colors, as shown in Figure 6 . The partial order induced on the 6 colors is the dual of the poset of Figure 1 .

Corollary 7 shows that we can obtain each minor poset as a "poset of colors", where the order on the colors is induced by a suitable coloring of a partition lattice. Therefore, our main goal is to characterize those colorings that can arise from a function. We define an abstract property of colorings of partition lattices, called admissibility (see Definition 11), and in Corollary 18 we prove that admissibility is indeed a necessary and sufficient condition for the existence of a function $f$ such that the given coloring is induced by $f$ (as in Corollary 7).

Definition 9. Let $c: \Pi_{n} \rightarrow C$ be a coloring, and let $\alpha, \beta \in \Pi_{n}$. We write $\alpha \sim \beta$ if the intervals $[\alpha, \top]$ and $[\beta, \top]$ are isomorphic as colored posets, i.e., there is an isomorphism $\varphi$ from $[\alpha, \top]$ to $[\beta, \top]$ such that $c(\xi)=c(\varphi(\xi))$ for all $\xi \in[\alpha, \top]$.

Proposition 10. Let $c: \Pi_{n} \rightarrow C$ be a coloring, and let $\alpha, \vartheta \in \Pi_{n}$ such that $\alpha \leq \vartheta \prec$ $\top$. Then the following two conditions are equivalent.

(i) For every $\gamma \in \Pi_{n}$ with $\alpha \prec \gamma \not \leq \vartheta$, the map

$$
\varphi_{\gamma}:[\alpha, \vartheta] \rightarrow[\gamma, \top], \xi \mapsto \xi \vee \gamma
$$

is a color-preserving isomorphism (cf. Fact 3 and see also Figure 3).

(ii) One of the blocks of $\alpha$ is also a block of $\vartheta$ and

$$
\forall \xi \in[\alpha, \top]: c(\xi)=c(\xi \wedge \vartheta) .
$$

Proof. Observe that if $\alpha=\vartheta$, then the equivalence of (i) and (ii) is obvious: both are equivalent to $c(\alpha)=c(T)$ (in this case the partition $\gamma$ in (i) must be $T$ ). Therefore, we may assume that $\alpha<\vartheta$, as depicted in Figure 3 . Then $\alpha$ has at least three blocks, and there exist partitions $\gamma \neq \top$ with $\alpha \prec \gamma \not \leq \vartheta$ (just merge any two blocks of $\alpha$ that are not merged in $\vartheta$ ).

Assume first that (i) holds. If $k=|\alpha|$ and the two blocks of $\vartheta$ are unions of $s$ and $k-s$ blocks of $\alpha$, respectively, then $[\alpha, \vartheta] \cong \Pi_{s} \times \Pi_{k-s}$ and $[\gamma, \top] \cong \Pi_{k-1}$. By condition (i), these two lattices are isomorphic: $\Pi_{s} \times \Pi_{k-s} \cong \Pi_{k-1}$. Partition 


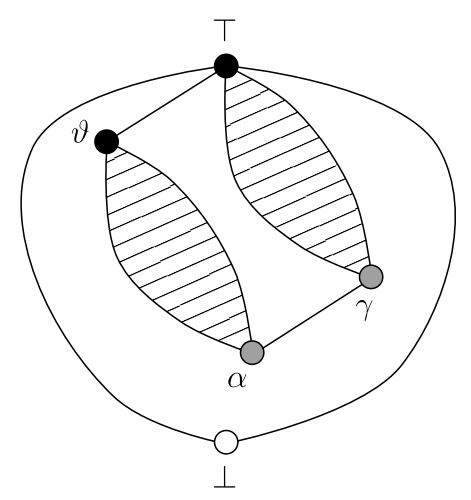

Figure 3. The intervals $[\alpha, \vartheta]$ and $[\gamma, \top]$ are isomorphic as colored posets.

lattices are simple (see Ore [8, Theorem 8] and Beran, Ježek [1, Theorem 1]), hence directly indecomposable; therefore, we must have $s=1$ or $s=k-1$. Thus one of the blocks of $\alpha$ is indeed a block of $\vartheta$, i.e., $\alpha$ and $\vartheta$ are of the form $\alpha=\left\{V_{1}, \ldots, V_{k}\right\}$ and $\vartheta=\left\{V_{1}, V_{2} \cup \cdots \cup V_{k}\right\}$. To prove (1), let us fix an arbitrary partition $\xi \in[\alpha, \top]$. If $\xi \leq \vartheta$, then $\xi \wedge \vartheta=\xi$, hence (1) holds trivially. If $\xi \not \leq \vartheta$, then $V_{1}$ is merged with at least one other block of $\alpha$ in $\xi$; we can suppose without loss of generality that $V_{1} \cup V_{2}$ is contained in a block of $\xi$. Letting $\gamma=\left\{V_{1} \cup V_{2}, V_{3}, \ldots, V_{k}\right\}$, we see that $\xi \in[\gamma, \top]$ and $\alpha \prec \gamma \not \leq \vartheta$, thus $c\left(\varphi_{\gamma}^{-1}(\xi)\right)=c(\xi)$ by (i). Recall from Fact 3 that $\varphi_{\gamma}^{-1}(\xi)=\xi \wedge \vartheta$, hence $c(\xi \wedge \vartheta)=c\left(\varphi_{\gamma}^{-1}(\xi)\right)=c(\xi)$, which completes the proof of (ii).

Now assume that (ii) holds. If $\alpha \prec \gamma \not \leq \vartheta$ and $\xi \in[\alpha, \vartheta]$, then replacing $\xi$ by $\xi \vee \gamma$ in (1) we obtain

$$
c(\xi \vee \gamma)=c((\xi \vee \gamma) \wedge \vartheta)=c(\xi),
$$

which proves that $\varphi_{\gamma}$ preserves colors. (Here we used Fact 3 in the form $(\xi \vee \gamma) \wedge \vartheta=$ $\xi$.

Definition 11. Let $c: \Pi_{n} \rightarrow C$ be a coloring, and let $\alpha, \beta \in \Pi_{n}$. We write $\alpha \rightsquigarrow_{1} \beta$ if $\alpha \prec \beta$ and there is a partition $\vartheta \in \Pi_{n}$ with $\alpha \leq \vartheta \prec \top$ and $\beta \not \leq \vartheta$ such that the equivalent conditions of Proposition 10 are satisfied (in particular, condition (ii) holds with $\gamma=\beta$ ).

Let $\rightsquigarrow$ be the reflexive-transitive closure of $\rightsquigarrow 1$, i.e., $\alpha \rightsquigarrow \beta$ if and only if there exist $\alpha_{0}, \ldots, \alpha_{k} \in \Pi_{n}$ for some $k \geq 0$ such that $\alpha=\alpha_{0} \rightsquigarrow_{1} \alpha_{1} \rightsquigarrow_{1} \cdots \rightsquigarrow_{1} \alpha_{k}=\beta$ (this includes the case $\alpha=\beta$ when $k=0$ ).

We say that the coloring $c$ is admissible, if for all $\alpha, \beta \in \Pi_{n}$, we have

$$
c(\alpha)=c(\beta) \Longrightarrow \exists \alpha^{\prime}, \beta^{\prime} \in \Pi_{n}: \alpha \rightsquigarrow \alpha^{\prime} \sim \beta^{\prime} \rightsquigarrow \beta .
$$

Remark 12. Note that if $\alpha \sim \beta$ or $\alpha \rightsquigarrow \beta$, then $c(\alpha)=c(\beta)$. Thus the reverse implication of (2) always holds.

Proposition 13. Let $f: A^{n} \rightarrow B$ be an arbitrary function, and let $c(\alpha)=f_{\alpha} / \equiv$ for all $\alpha \in \Pi_{n}$. Then $c$ is an admissible coloring of $\Pi_{n}$.

Proof. Let $\alpha=\left\{V_{1}, \ldots, V_{k}\right\} \in \Pi_{n}$ be an arbitrary partition of size $k \geq 2$, and assume that $V_{1}$ is an inessential variable of $f_{\alpha}$. Let $\vartheta=\left\{V_{1}, V_{2} \cup \cdots \cup V_{k}\right\}$ and suppose that $\alpha \prec \gamma \not \leq \vartheta$. Clearly, $\gamma$ is obtained from $\alpha$ by merging $V_{1}$ with another block $V_{j}$. This means that we get $f_{\gamma}$ from $f_{\alpha}$ by identifying the inessential variable $V_{1}$ with another variable, hence we have $f_{\alpha} \equiv f_{\gamma}$, that is $c(\alpha)=c(\gamma)$. Similarly, for any $\xi \in[\alpha, \vartheta]$, denoting by $\varphi(\xi)=\xi \vee \gamma$ the partition obtained from $\xi$ by merging $V_{1}$ (which must be a block of $\xi$ ) with the block containing $V_{j}$, we have $c(\xi)=c(\varphi(\xi))$, therefore condition (i) of Proposition 10 is satisfied. Thus we can conclude that $\alpha \rightsquigarrow_{1} \gamma$ for every $\gamma \in \Pi_{n}$ such that $\alpha \prec \gamma \not \leq \vartheta$.

We have proved that if $f_{\alpha}$ has an inessential variable, then there exists an upper cover $\gamma$ of $\alpha$ such that $\alpha \rightsquigarrow 1 \gamma$. Proceeding this way (always identifying an inessential 
variable with another variable as long as there is an inessential variable), we finally arrive at a partition $\alpha^{\prime}$ such that $\alpha \rightsquigarrow \alpha^{\prime}$ and all variables of $f_{\alpha^{\prime}}$ are essential.

Now we are ready to prove that $c$ is an admissible coloring. Assume that $c(\alpha)=$ $c(\beta)$, i.e., $f_{\alpha} \equiv f_{\beta}$, and use the above procedure to find partitions $\alpha^{\prime}$ and $\beta^{\prime}$ such that $\alpha \rightsquigarrow \alpha^{\prime}$ and $\beta \rightsquigarrow \beta^{\prime}$ with $f_{\alpha^{\prime}}$ and $f_{\beta^{\prime}}$ depending on all their variables. Since $f_{\alpha^{\prime}} \equiv f_{\alpha} \equiv f_{\beta} \equiv f_{\beta^{\prime}}$, the functions $f_{\alpha^{\prime}}$ and $f_{\beta^{\prime}}$ are equivalent, and this implies that they can be obtained from each other by permuting (renaming) the variables, since both functions have only essential variables. This permutation of variables induces naturally a color-preserving isomorphism between the intervals $\left[\alpha^{\prime}, \top\right]$ and $\left[\beta^{\prime}, \top\right]$, showing that $\alpha^{\prime} \sim \beta^{\prime}$. Thus we have $\alpha \rightsquigarrow \alpha^{\prime} \sim \beta^{\prime} \leftarrow \beta$, and this proves that (2) is satisfied.

Remark 14. If $f$ depends on all of its variables, then $c(\perp)=f / \equiv$ appears only at $\perp$ in the coloring of Proposition 13 . Therefore, one may always assume without loss of generality that $\perp$ is the unique element of $\Pi_{n}$ with color $c(\perp)$. On the other hand, one cannot assume the same about the color of $T$ : a function can have several minors that are equivalent to $f_{\top}=f(x, \ldots, x)$ (see also Remark 22 .

Next we would like to prove the following converse of Proposition 13 , for any admissible coloring $c: \Pi_{n} \rightarrow C$, there is a function $f: A^{n} \rightarrow B$ such that two partitions of $[n]$ have the same color if and only if the corresponding minors of $f$ are equivalent. To construct this function, let $A$ be any set with at least $n$ elements, let $B=C$, and define $f: A^{n} \rightarrow B$ by $f(\mathbf{a}):=c($ ker $\mathbf{a})$ for all $\mathbf{a} \in A^{n}$. Here ker a denotes the (partition corresponding to the) kernel of the map a: $[n] \rightarrow A, i \mapsto a_{i}$. All partitions of $[n]$ with at most $|A|$ blocks arise in the form ker a, therefore our assumption $|A| \geq n$ guarantees that in fact every element of $\Pi_{n}$ will occur. We will show in Theorem 17 that the above function has the desired property, thus we can conclude that every poset that appears as the poset of minors of a function can be represented by a function $f$ having the special property that $f(\mathbf{a})$ is determined by the kernel of $\mathbf{a}$.

Let $f$ be the function defined above, and let us consider an arbitrary minor $f_{\alpha}$. From the definition of a minor we have that $f_{\alpha}(\mathbf{a})=f\left(\mathbf{a} \circ \operatorname{nat}_{\alpha}\right)=c\left(\operatorname{ker}\left(\mathbf{a} \circ\right.\right.$ nat $\left.\left._{\alpha}\right)\right)$ for all $\mathbf{a} \in A^{\alpha}$. Observe that the partition $\operatorname{ker}\left(\mathbf{a} \circ\right.$ nat $\left._{\alpha}\right)$ is a coarsening of $\alpha$ (merging two blocks of $\alpha$ if and only if a assigns the same value to them). Moreover, the assumption $|A| \geq n$ ensures that we obtain every coarsening of $\alpha$ (every element of the interval $[\alpha, \top])$ this way. This observation will be of key importance in the next two lemmas, which prepare the ground for the proof of Theorem 17, our main result in this section.

Lemma 15. Let $c: \Pi_{n} \rightarrow C$ be an arbitrary coloring, and let the function $f: A^{n} \rightarrow C$ be defined by $f(\mathbf{a})=c(\operatorname{ker} \mathbf{a})$ for all $\mathbf{a} \in A^{n}$, where $A$ is a finite set with at least $n$ elements. For arbitrary partitions $\alpha, \beta \in \Pi_{n}$, the minors $f_{\alpha}$ and $f_{\beta}$ can be obtained from each other by a permutation of variables if and only if $\alpha \sim \beta$.

Proof. First let us assume that $f_{\alpha}$ and $f_{\beta}$ can be obtained from each other by a permutation of variables. This means that $\alpha$ and $\beta$ have the same number of blocks, and there is a bijection $\pi: \beta \rightarrow \alpha$ such that $f_{\alpha}(\mathbf{a})=f_{\beta}(\mathbf{a} \circ \pi)$ for all $\mathbf{a} \in A^{\alpha}$. By the definition of a minor, we can rewrite this equality as $f\left(\mathbf{a} \circ\right.$ nat $\left._{\alpha}\right)=f\left(\mathbf{a} \circ \pi \circ\right.$ nat $\left._{\beta}\right)$, which in turn can be formulated as $c\left(\operatorname{ker}\left(\mathbf{a} \circ \operatorname{nat}_{\alpha}\right)\right)=c\left(\operatorname{ker}\left(\mathbf{a} \circ \pi \circ \operatorname{nat}_{\beta}\right)\right)$. The partition $\operatorname{ker}\left(\mathbf{a} \circ\right.$ nat $\left._{\alpha}\right)$ is a coarsening of $\alpha$, and $\operatorname{ker}\left(\mathbf{a} \circ \pi \circ\right.$ nat $\left._{\beta}\right)$ is a coarsening of $\beta$, which can be obtained from $\beta$ by merging two blocks if and only if the images of these two blocks under $\pi$ are merged in $\operatorname{ker}\left(\mathbf{a} \circ\right.$ nat $\left._{\alpha}\right)$. Since all coarsenings of $\alpha$ and $\beta$ appear here, we obtain a color-preserving isomorphism

$$
\tilde{\pi}:[\alpha, \top] \rightarrow[\beta, \top], \operatorname{ker}\left(\mathbf{a} \circ \operatorname{nat}_{\alpha}\right) \mapsto \operatorname{ker}\left(\mathbf{a} \circ \pi \circ \operatorname{nat}_{\beta}\right),
$$

showing that $\alpha \sim \beta$.

Next assume that $\alpha \sim \beta$, i.e., that there is a color-preserving isomorphism $\varphi:[\alpha, \top] \rightarrow$ $[\beta, \top]$. Since $[\alpha, \top] \cong \Pi_{\alpha}$ and $[\beta, \top] \cong \Pi_{\beta}$, Theorem 2 implies that $\varphi$ is induced by a 
bijection $\pi: \beta \rightarrow \alpha$. From the above considerations it follows that $\varphi$ is exactly the isomorphism $\tilde{\pi}$ defined by (3), and, since $\varphi$ is a color-preserving isomorphism, this means that $c\left(\operatorname{ker}\left(\mathbf{a} \circ\right.\right.$ nat $\left.\left._{\alpha}\right)\right)=c\left(\operatorname{ker}\left(\mathbf{a} \circ \pi \circ\right.\right.$ nat $\left.\left._{\beta}\right)\right)$ for all $\mathbf{a} \in A^{\alpha}$. By the definition of $f$, we conclude $f_{\alpha}(\mathbf{a})=f_{\beta}(\mathbf{a} \circ \pi)$, hence $f_{\alpha}$ can be obtained from $f_{\beta}$ by a permutation of variables.

Lemma 16. Let $c: \Pi_{n} \rightarrow C$ be an arbitrary coloring, and let the function $f: A^{n} \rightarrow C$ be defined by $f(\mathbf{a})=c($ ker $\mathbf{a})$ for all $\mathbf{a} \in A^{n}$, where $A$ is a finite set with at least $n$ elements. For arbitrary partitions $\alpha, \beta \in \Pi_{n}$, the minor $f_{\beta}$ can be obtained from $f_{\alpha}$ by identifying an inessential variable with another variable if and only if $\alpha \rightsquigarrow_{1} \beta$.

Proof. To prove the "only if" part of the lemma, let $\alpha=\left\{V_{1}, \ldots, V_{k}\right\} \in \Pi_{n}$ and let us assume (without loss of generality) that the first variable of $f_{\alpha}$ is inessential and that $f_{\beta}$ is obtained from $f_{\alpha}$ by identifying the first two variables. Then we have $\beta=\left\{V_{1} \cup V_{2}, V_{3}, \ldots, V_{k}\right\}$, and let us put $\vartheta=\left\{V_{1}, V_{2} \cup \cdots \cup V_{k}\right\}$. Clearly, $\alpha \leq \vartheta \prec \top$ and $\alpha \prec \beta \not \leq \vartheta$, so we only need to verify $(1)$. Let $\xi=\left\{W_{1}, \ldots, W_{\ell}\right\} \in[\alpha, \top]$ be an arbitrary partition, and let $W_{1}$ be the block of $\xi$ that contains $V_{1}$. If $W_{1}=$ $V_{1}$ then $\xi \leq \vartheta$, hence $\xi=\xi \wedge \vartheta$, and then (1) holds trivially. If $W_{1} \subset V_{1}$, then $\xi \wedge \vartheta=\left\{V_{1}, W_{1} \backslash V_{1}, W_{2}, \ldots, W_{\ell}\right\}$ and $V_{1}$ is an inessential variable in $f_{\xi \wedge \vartheta}$. Therefore, if $a_{0}, \ldots, a_{\ell} \in A$ are pairwise different (such elements exist, as $|A| \geq n$ ), then we have

$$
c(\xi \wedge \vartheta)=f_{\xi \wedge \vartheta}\left(a_{0}, a_{1}, a_{2}, \ldots, a_{\ell}\right)=f_{\xi \wedge \vartheta}\left(a_{1}, a_{1}, a_{2}, \ldots, a_{\ell}\right)=c(\xi),
$$

thus (1) holds. This proves that $\alpha \rightsquigarrow_{1} \beta$, as claimed.

For the "if" part, assume that $\alpha \rightsquigarrow_{1} \beta$. We may suppose (without loss of generality) that $\alpha=\left\{V_{1}, \ldots, V_{k}\right\}, \beta=\left\{V_{1} \cup V_{2}, V_{3}, \ldots, V_{k}\right\}$ and the partition $\vartheta$ justifying $\alpha \rightsquigarrow 1 \beta$ according to Definition 11 is $\vartheta=\left\{V_{1}, V_{2} \cup \cdots \cup V_{k}\right\}$. Let a $\in A^{\alpha}$ and let $\xi=\operatorname{ker}\left(\mathbf{a} \circ\right.$ nat $\left._{\alpha}\right)$. Then we have $f_{\alpha}\left(a_{1}, \ldots, a_{k}\right)=c(\xi)$ and $f_{\beta}\left(a_{2}, \ldots, a_{k}\right)=$ $f_{\alpha}\left(a_{2}, a_{2}, \ldots, a_{k}\right)=c\left(\operatorname{ker}\left(\left(a_{2}, a_{2}, \ldots, a_{k}\right) \circ\right.\right.$ nat $\left.\left._{\alpha}\right)\right)$. Since

$$
\operatorname{ker}\left(\left(a_{2}, a_{2}, \ldots, a_{k}\right) \circ \operatorname{nat}_{\alpha}\right)=\left(\operatorname{ker}\left(\left(a_{1}, a_{2}, \ldots, a_{k}\right) \circ \text { nat }_{\alpha}\right) \wedge \vartheta\right) \vee \beta=(\xi \wedge \vartheta) \vee \beta,
$$

we see that $f_{\beta}\left(a_{2}, \ldots, a_{k}\right)=c((\xi \wedge \vartheta) \vee \beta)$.

From $\alpha \rightsquigarrow 1 \beta$ it follows that $c(\xi)=c(\xi \wedge \vartheta)$ (using condition (ii) of Proposition 10 and $c(\xi \wedge \vartheta)=c((\xi \wedge \vartheta) \vee \beta)$ (using condition (i) of Proposition 10 with $\xi \wedge \vartheta$ in place of $\xi$ and $\beta$ in place of $\gamma)$. We can conclude that

$$
f_{\alpha}\left(a_{1}, a_{2}, \ldots, a_{k}\right)=c(\xi)=c((\xi \wedge \vartheta) \vee \beta)=f_{\beta}\left(a_{2}, \ldots, a_{k}\right)
$$

for all $a_{1}, a_{2}, \ldots, a_{k} \in A$. This means that the first variable of $f_{\alpha}$ is inessential, and $f_{\beta}$ is obtained by identifying this inessential variable with the second variable.

With the help of the previous two lemmas we can now prove that every admissible coloring of $\Pi_{n}$ can be realized by minors of an $n$-variable function.

Theorem 17. Let $c: \Pi_{n} \rightarrow C$ be an admissible coloring, and let the function $f: A^{n} \rightarrow$ $C$ be defined by $f(\mathbf{a})=c(\operatorname{ker} \mathbf{a})$ for all $\mathbf{a} \in A^{n}$, where $A$ is a finite set with at least $n$ elements. Then for every $\alpha, \beta \in \Pi_{n}$, we have $f_{\alpha} \equiv f_{\beta}$ if and only if $c(\alpha)=c(\beta)$.

Proof. Suppose first that $f_{\alpha} \equiv f_{\beta}$. Let $\alpha=\left\{V_{1}, \ldots, V_{k}\right\} \in \Pi_{n}$ and assume that $V_{1}, \ldots, V_{\ell}$ are inessential variables and $V_{\ell+1}, \ldots, V_{k}$ are essential variables in $f_{\alpha}$. If $\alpha^{\prime}=\left\{V_{1} \cup \cdots \cup V_{\ell} \cup V_{\ell+1}, V_{\ell+2}, \ldots, V_{k}\right\}$, then $f_{\alpha^{\prime}}$ depends on all of its variables, and $f_{\alpha^{\prime}}$ can be obtained from $f_{\alpha}$ by repeatedly identifying an inessential variable with an essential one. Similarly, let $f_{\beta^{\prime}}$ be the "essential minor" of $f_{\beta}$. Clearly, $f_{\alpha} \equiv f_{\beta}$ implies that $f_{\alpha^{\prime}}$ and $f_{\beta^{\prime}}$ can be obtained from each other by a permutation of variables. Now Lemma 15 and Lemma 16 yield $\alpha \rightsquigarrow \alpha^{\prime} \sim \beta^{\prime} \leftrightarrow \beta$, and then $c(\alpha)=c(\beta)$ follows (see Remark 12 .

Conversely, if $c(\alpha)=c(\beta)$, then, by the admissibility of the coloring $c$, there exist $\alpha^{\prime}, \beta^{\prime} \in \Pi_{n}$ such that $\alpha \rightsquigarrow \alpha^{\prime} \sim \beta^{\prime} \sim \beta$. Lemma 16 shows that $f_{\alpha} \equiv f_{\alpha^{\prime}}$ and $f_{\beta} \equiv f_{\beta^{\prime}}$, and Lemma 15 shows that $f_{\alpha^{\prime}} \equiv f_{\beta^{\prime}}$. Therefore, we can conclude that $f_{\alpha}$ and $f_{\beta}$ are equivalent. 
Proposition 13 and Theorem 17 together yield the following characterization of minor posets.

Corollary 18. A poset $P$ belongs to $\mathcal{M}$ (i.e., isomorphic to the poset of all minors of some function $f$ ) if and only if there is an admissible coloring $c: \Pi_{n} \rightarrow C$ for some natural number $n$ and for some nonempty set $C$ such that $P^{d} \cong \Pi_{n} /$ ker $c$.

\section{Constructions And examples}

In this section we give some (families) of examples of minor posets, and we also present some constructions which allow us to build new minor posets from known ones. We denote the poset of integer partitions of $n$ by $P_{n}$ (see Birkhoff 2 for the definition of this poset).

Theorem 19. The following are minor posets for all natural numbers $m$ and $n$ :

(i) the dual of the partition lattice $\Pi_{n}$;

(ii) the dual of the poset $P_{n}$ of integer partitions of $n$;

(iii) the n-element chain $\mathbf{n}$;

(iv) the lattice $\mathbf{m} \oplus M_{n}$;

(v) the $n$-dimensional cube (Boolean lattice) $\mathbf{2}^{n}$.

Proof. In each case we give an admissible coloring of a partition lattice such that the corresponding quotient is dually isomorphic to the desired poset. Except for (v), we leave it to the reader to verify that these colorings are indeed admissible and that they yield the desired quotient.

(i) If $c: \Pi_{n} \rightarrow \Pi_{n}$ is the identity map, then clearly $\Pi_{n} /$ ker $c$ is dually isomorphic to $\Pi_{n}^{d}$.

(ii) For $\alpha=\left\{V_{1}, \ldots, V_{k}\right\} \in \Pi_{n}$, let $c(\alpha)$ be the integer partition $n=\left|V_{1}\right|+\cdots+\left|V_{k}\right|$ given by the sizes of the blocks of $\alpha$. Then $c: \Pi_{n} \rightarrow P_{n}$ is an admissible coloring and $\Pi_{n} / \operatorname{ker} c$ is isomorphic to $P_{n}$.

(iii) For the coloring $c: \Pi_{n} \rightarrow[n], \alpha \mapsto|\alpha|$, the quotient $\Pi_{n} / \operatorname{ker} c$ is (the dual of) an $n$-element chain.

(iv) Choose a natural number $k$ such that $k \geq m+3$ and $\left(\begin{array}{l}k \\ 2\end{array}\right) \geq n$. Let $c_{1}, \ldots, c_{n}$ and $d_{1}, \ldots, d_{m}$ be pairwise distinct colors, all distinct from white and black, and let us color $\Pi_{k}$ as follows. The bottom element of $\Pi_{k}$ is white. The atoms receive colors $c_{1}, \ldots, c_{n}$ in an arbitrary way so that all these $n$ colors are used. For $i=1, \ldots, m$, the partitions with exactly $k-i-1$ blocks receive color $d_{i}$. All remaining partitions, i.e., the ones with at most $k-m-2$ blocks are black (see Figure 7 with $m=1, n=3, k=4$ ). Then $\Pi_{k} / \operatorname{ker} c$ is isomorphic to $M_{n} \oplus \mathbf{m}$, hence dually isomorphic to $\mathbf{m} \oplus M_{n}$.

(v) Let $c$ be the coloring that assigns to every partition $\alpha=\left\{V_{1}, \ldots, V_{k}\right\} \in \Pi_{n+1}$ the set of minimal elements of the blocks of $\alpha$ (under the natural ordering $1<\cdots<n+1)$, that is, $c(\alpha):=\left\{\min V_{1}, \ldots, \min V_{k}\right\}$. Since $|c(\alpha)|=$ $|\alpha|$, only partitions on the same level of $\Pi_{n+1}$ can receive the same color. Therefore, in order to prove that $c$ is admissible, it suffices to show that $[\alpha, \top]$ and $[\beta, \top]$ are isomorphic as colored posets whenever $c(\alpha)=c(\beta)$. Let us consider the natural isomorphisms $\varphi:[\alpha, \top] \rightarrow \Pi_{\alpha}$ and $\psi:[\beta, \top] \rightarrow \Pi_{\beta}$. If $c(\alpha)=c(\beta)$, then for each block $V$ of $\alpha$, there exists a unique block $\pi(V)$ of $\beta$ such that $\min V=\min \pi(V)$, and this defines a bijection $\pi: \alpha \rightarrow \beta$. Now the composition $\psi^{-1} \circ \tilde{\pi} \circ \varphi$ is a color-preserving isomorphism from $[\alpha, \top]$ to $[\beta, \top]$.

To determine the quotient poset, observe that the image of $c$ consists of those subsets of $[n+1]$ that contain the element 1 , and we have $\alpha>\beta \Longrightarrow$ $c(\alpha) \subset c(\beta)$. Moreover, if $1 \in M \subset N \subseteq[n+1]$, then one can find partitions $\alpha, \beta \in \Pi_{n+1}$ with $\alpha>\beta$ and $c(\alpha)=M, c(\beta)=N$. This implies that $\Pi_{n+1} / \operatorname{ker} c$ is isomorphic to the lattice of subsets of $[n+1]$ containing 1 , which is (dually) isomorphic to $\mathbf{2}^{n}$. Figure 8 illustrates the coloring and the corresponding quotient for $n=3$. 
Remark 20. For the first three items of Theorem 19, it is easy to find functions that realize the given posets. If $f$ is an injective $n$-variable function, then no two minors of $f$ are equivalent, hence the poset of minors of $f$ is isomorphic to the dual of the partition lattice $\Pi_{n}$. For the second item, let us consider the function $f\left(x_{1}, \ldots, x_{n}\right)=$ $x_{1}+\cdots+x_{n}$ over the real numbers. If $\alpha \in \Pi_{n}$ has blocks of sizes $m_{1}, \ldots, m_{k}$ (hence $m_{1}+\cdots+m_{k}=n$ ), then $f_{\alpha}$ is equivalent to the function $m_{1} x_{1}+\cdots+m_{k} x_{k}$. This shows that the poset of minors of $f$ is isomorphic to the dual of the lattice of integer partitions of $n$. Finally, let us consider the function $f\left(x_{1}, \ldots, x_{n}\right)=x_{1} \vee \cdots \vee x_{n}$, where $\vee$ is a semilattice operation. If $\alpha \in \Pi_{n}$ has $k$ blocks, then $f_{\alpha}$ is equivalent to the function $x_{1} \vee \cdots \vee x_{k}$. This implies that the poset of minors of $f$ is an $n$-element chain.

Proposition 21. The class $\mathcal{M}$ is closed under taking principal ideals: if $P \in \mathcal{M}$ and $a \in P$, then the principal ideal $\left[\perp_{P}, a\right]$ is also a member of $\mathcal{M}$.

Proof. If $f$ is a function such that $\downarrow f$ is isomorphic to $P$, and $a \in P$, then $f$ has a minor $f_{\alpha}$ corresponding to $a$, and $\downarrow f_{\alpha}$ is isomorphic to the principal ideal $\left[\perp_{P}, a\right]$.

Remark 22. A natural idea to prove an analogous statement for principal filters would be the following. Take an admissible coloring $c$ of $\Pi_{n}$ such that $\Pi_{n} / \operatorname{ker} c \cong P^{d}$, and let $\alpha$ correspond to $a$ under this isomorphism. Change all colors outside of $\downarrow \alpha$ to the color of $\alpha$; then the resulting quotient poset of $\Pi_{n}$ will be dually isomorphic to the principal filter $\left[a, \top_{P}\right]$ of $P$. However, unfortunately, this modified coloring will not be admissible in general (a counterexample is provided in Online Resources 1 and 2 ). Nevertheless, it might be still true that $\mathcal{M}$ is closed under taking principal filters, but a different argument would be needed to prove this.

In the following theorem we prove that one can always add a new top element to a minor poset. Recalling that the ordinal sum of posets is denoted by $\oplus$, the poset obtained by adding a new top element to $P$ can be written as $P \oplus \mathbf{1}$.

Theorem 23. If $P \in \mathcal{M}$, then $P \oplus \mathbf{1} \in \mathcal{M}$.

Proof. By Corollary 18, there is an admissible coloring $c: \Pi_{n-1} \rightarrow C$ for some natural number $n$ and for some nonempty set $C$ such that $\Pi_{n-1} / \operatorname{ker} c \cong P^{d}$. For any $\xi \in$ $\Pi_{n}$, let us simply write $\xi-n$ for the partition that is obtained from $\xi$ by deleting the element $n$. More precisely, if $\xi=\left\{V_{1}, \ldots, V_{k}\right\}$, and, say, $n \in V_{k}$, then let $\xi-$ $n=\left\{V_{1}, \ldots, V_{k} \backslash\{n\}\right\} \in \Pi_{n-1}$, discarding the block $V_{k} \backslash\{n\}$ if it is empty. Define $c^{*}: \Pi_{n} \rightarrow C$ by $c^{*}(\xi)=c(\xi-n)$. Later we will modify this coloring to obtain the poset $P \oplus \mathbf{1}$, but first let us check that $c^{*}$ is admissible. We shall need the following two observations.

1. If $\alpha \in \Pi_{n}$ and $\{n\} \notin \alpha$ (i.e., $n$ does not form a singleton block in $\alpha$ ), then the intervals $[\alpha, \top] \subseteq \Pi_{n}$ and $[\alpha-n, \top] \subseteq \Pi_{n-1}$ are isomorphic as colored posets under the map $\xi \mapsto \xi-n$.

2. For every $\alpha \in \Pi_{n}$ there exists $\alpha^{\prime} \in \Pi_{n}$ such that $\alpha \rightsquigarrow \alpha^{\prime}$ and $\{n\} \notin \alpha^{\prime}$. If $\{n\} \notin \alpha$, then we may choose $\alpha^{\prime}=\alpha$. Otherwise $\alpha$ is of the form $\alpha=\left\{V_{1}, \ldots, V_{k},\{n\}\right\}$. In this case let $\alpha^{\prime}=\left\{V_{1}, \ldots, V_{k} \cup\{n\}\right\}$ and $\vartheta=$ $\{\{1, \ldots, n-1\},\{n\}\}$. Then condition (ii) of Proposition 10 is satisfied, since $\xi-n=(\xi \wedge \vartheta)-n$ holds for all $\xi \in[\alpha, \top]$ (in fact, for all $\xi \in \Pi_{n}$ ), and therefore

$$
c^{*}(\xi)=c(\xi-n)=c((\xi \wedge \vartheta)-n)=c^{*}(\xi \wedge \vartheta) .
$$

This shows that $\alpha \rightsquigarrow 1 \alpha^{\prime}$.

Now we are ready to verify the admissibility of $c^{*}$. Assume that $\alpha, \beta \in \Pi_{n}$ and $c^{*}(\alpha)=c^{*}(\beta)$. By our second observation above, there exist $\alpha^{\prime}, \beta^{\prime} \in \Pi_{n}$ such that $\alpha \rightsquigarrow \alpha^{\prime}, \beta \rightsquigarrow \beta^{\prime}$ and $\{n\} \notin \alpha^{\prime}, \beta^{\prime}$. From Remark 12 we see that $c^{*}\left(\alpha^{\prime}\right)=c^{*}(\alpha)=$ $c^{*}(\beta)=c^{*}\left(\beta^{\prime}\right)$, and this implies $c\left(\alpha^{\prime}-n\right)=c\left(\beta^{\prime}-n\right)$. Since $c$ is an admissible coloring of $\Pi_{n-1}$, there exist $\gamma, \delta \in \Pi_{n-1}$ with $\alpha^{\prime}-n \rightsquigarrow \gamma \sim \delta \leftrightarrow \beta^{\prime}-n$. The partition $\alpha^{\prime}-n$ is obtained from $\alpha^{\prime}$ by removing $n$ from the block that contains $n$, and $\gamma$ is a 


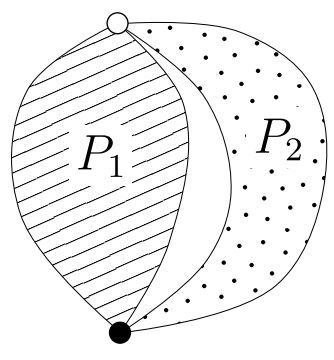

Figure 4 . The poset $P_{1} * P_{2}$.

coarsening of $\alpha^{\prime}-n$. Let us add the element $n$ to the block of $\gamma$ that contains the block of $\alpha^{\prime}$ from which $n$ was removed. This way we get a partition $\gamma^{\prime} \in \Pi_{n}$ with the properties $\gamma^{\prime} \geq \alpha^{\prime}$ and $\gamma^{\prime}-n=\gamma$. According to our first observation, the colored intervals $\left[\alpha^{\prime}, \top\right] \subseteq \Pi_{n}$ and $\left[\alpha^{\prime}-n, \top\right] \subseteq \Pi_{n-1}$ are isomorphic, thus $\alpha^{\prime}-n \rightsquigarrow \gamma$ implies $\alpha^{\prime} \rightsquigarrow \gamma^{\prime}$, and then $\alpha \rightsquigarrow \gamma^{\prime}$ follows, as $\alpha \rightsquigarrow \alpha^{\prime}$. In a similar way we can construct a partition $\delta^{\prime} \in \Pi_{n}$ such that $\delta^{\prime}-n=\delta$ and $\beta \rightsquigarrow \delta^{\prime}$. Using our first observation again, and taking into account that $\gamma \sim \delta$, we find the isomorphisms $\left[\gamma^{\prime}, \top\right] \cong[\gamma, \top] \cong$ $[\delta, \top] \cong\left[\delta^{\prime}, \top\right]$ (all of the underlying isomorphisms are color preserving). This means that $\alpha \rightsquigarrow \gamma^{\prime} \sim \delta^{\prime} \sim \beta$, hence (2) holds, and therefore $c^{*}$ is indeed admissible.

The quotient poset $\Pi_{n} / \operatorname{ker} c^{*}$ is isomorphic to $P^{d}$. To see this, note that $c^{*}$ uses the same colors as $c$; moreover, for any two colors $c_{1}, c_{2} \in C$ the following two conditions are equivalent:

(a) $\exists \xi_{1}, \xi_{2} \in \Pi_{n}^{d}: \quad \xi_{1} \leq \xi_{2} \quad$ and $\quad c^{*}\left(\xi_{1}\right)=c_{1}, c^{*}\left(\xi_{2}\right)=c_{2}$;

(b) $\exists \eta_{1}, \eta_{2} \in \Pi_{n-1}^{d}: \quad \eta_{1} \leq \eta_{2} \quad$ and $\quad c\left(\eta_{1}\right)=c_{1}, \quad c\left(\eta_{2}\right)=c_{2}$.

Indeed, for (a) $\Longrightarrow$ (b) set $\eta_{i}=\xi_{i}-n$, and for (b) $\Longrightarrow$ (a) set $\xi_{i}=\eta_{i} \cup\{\{n\}\}$.

Now let us introduce a new color $* \notin C$ and modify the coloring $c^{*}$ by changing the color of $\perp$ to $*$ (the colors of the other elements remain the same). Clearly, this new coloring is also admissible, and the corresponding quotient of $\Pi_{n}$ is isomorphic to $\mathbf{1} \oplus P^{d}$ (note that the "old" color $c^{*}(\perp)$ does still appear, for instance as $c^{*}(\{\{1\},\{2\}, \ldots,\{n-1, n\}\})$. Therefore, $P \oplus \mathbf{1}$ (the dual of $\left.\mathbf{1} \oplus P^{d}\right)$ belongs to $\mathcal{M}$ by Corollary 18

Remark 24. It is a natural question whether $P \in \mathcal{M}$ implies $\mathbf{1} \oplus P \in \mathcal{M}$. A simple proof could be obtained by changing the color of $T$ to a new color $*$ at the end of the previous proof. Unfortunately, this new coloring is not necessarily admissible. (As an example, let us consider the coloring $c$ of $\Pi_{2}$ that colors the bottom element white and the top element black. The corresponding coloring $c^{*}$ assigns the color black to $\{\{1,2\},\{3\}\}$ and to $\{\{1,2,3\}\}$, and it assigns the color white to the remaining three elements of $\Pi_{3}$. Now if we change the color of the top element from black to a new color $*$, then the resulting coloring is no longer admissible.) Thus it remains an open problem whether adding a new bottom element to a minor poset yields a minor poset or not.

Next we describe a construction of "gluing" two posets together, and we show that $\mathcal{M}$ is closed under this construction. For finite bounded posets $P_{1}$ and $P_{2}$, let $P_{1} * P_{2}$ denote the poset obtained from the disjoint union (parallel sum) of $P_{1}$ and $P_{2}$ by identifying the top elements as well as the bottom elements (see Figure 4). Formally, we have

$$
P_{1} * P_{2}=\left(P_{1} \backslash\left\{\perp_{P_{1}}, \top_{P_{1}}\right\}\right) \cup\left(P_{2} \backslash\left\{\perp_{P_{2}}, \top_{P_{2}}\right\}\right) \cup\left\{\perp_{P_{1} * P_{2}}, \top_{P_{1} * P_{2}}\right\},
$$

where the ordering on $P_{i} \backslash\left\{\perp_{P_{i}}, \top_{P_{i}}\right\}$ is inherited from $P_{i}$ and there are no comparabilities between $P_{1} \backslash\left\{\perp_{P_{1}}, \top_{P_{1}}\right\}$ and $P_{2} \backslash\left\{\perp_{P_{2}}, \top_{P_{2}}\right\}$; furthermore, $\perp_{P_{1} * P_{2}}$ and $\top_{P_{1} * P_{2}}$ are the least and greatest elements of $P_{1} * P_{2}$.

Theorem 25. If $P_{1}, P_{2} \in \mathcal{M}$, then $P_{1} * P_{2} \in \mathcal{M}$. 
Proof. Suppose that $P_{1}, P_{2} \in \mathcal{M}$, and let $c_{i}: \Pi_{W_{i}} \rightarrow C_{i}$ be admissible colorings such that $\Pi_{W_{i}} / \operatorname{ker} c_{i} \cong P_{i}^{d}$ for $i=1,2$. We assume that the sets $W_{1}$ and $W_{2}$ are disjoint, and also that $c_{1}\left(\perp_{1}\right)=c_{2}\left(\perp_{2}\right)=\boldsymbol{\phi}, c_{1}\left(T_{1}\right)=c_{2}\left(T_{2}\right)=\varnothing$ but apart from these two colors, there is no common color used in $c_{1}$ and $c_{2}$. (Here $\perp_{i}$ and $\top_{i}$ denote the bottom and top elements of $\Pi_{W_{i}}$.) By Remark 14, we may also suppose that the color appears only at the bottom in $c_{1}$ as well as in $c_{2}$. We shall construct an admissible coloring $c: \Pi_{W} \rightarrow C$ with $W=W_{1} \cup W_{2}$ and $C=C_{1} \cup C_{2}$ such that $\Pi_{W} / \operatorname{ker} c \cong\left(P_{1} * P_{2}\right)^{d}$.

For $i=1,2$, let $\omega_{i} \in \Pi_{W}$ be the partition of $W$ whose only non-singleton block is $W_{i}$, and let $\iota_{i}: \Pi_{W_{i}} \rightarrow \Pi_{W}$ be the natural embedding that maps $\Pi_{W_{i}}$ isomorphically onto $\left[\perp, \omega_{i}\right]$. We define the desired coloring $c$ by

$$
c(\xi)= \begin{cases}c_{i}\left(\iota_{i}^{-1}(\xi)\right), & \text { if } \xi \in\left[\perp, \omega_{i}\right] \text { for some } i \in\{1,2\} ; \\ \nabla, & \text { if } \xi \notin\left[\perp, \omega_{1}\right] \cup\left[\perp, \omega_{2}\right] .\end{cases}
$$

Note that $c$ is well defined, as the intervals $\left[\perp, \omega_{1}\right]$ and $\left[\perp, \omega_{2}\right]$ intersect only at the bottom, and $c_{1}\left(\perp_{1}\right)=c_{2}\left(\perp_{2}\right)$. It is clear that $\Pi_{W} / \operatorname{ker} c \cong\left(P_{1} * P_{2}\right)^{d}$; we only need to verify that $c$ is admissible.

Let $\alpha, \beta \in \Pi_{W}$ such that $c(\alpha)=c(\beta)$. If $c(\alpha)=c(\beta)=\varnothing$ then 2 is clear, since $c^{-1}(\nabla)$ is an upset in $\Pi_{W}$. If this is not the case, then either both $\alpha$ and $\beta$ lie in $\left[\perp, \omega_{1}\right]$ or both are in $\left[\perp, \omega_{2}\right]$, as there is no common color in $C_{1}$ and $C_{2}$ except for $\varnothing$ and $\boldsymbol{\phi}$. In the following we will assume that $i=1$; the other case is essentially the same. Thus $c_{1}\left(\iota_{1}^{-1}(\alpha)\right)=c_{1}\left(\iota_{1}^{-1}(\beta)\right)$, and then we have

$$
\iota_{1}^{-1}(\alpha) \rightsquigarrow \alpha^{\prime} \sim \beta^{\prime} \rightsquigarrow \iota_{1}^{-1}(\beta)
$$

for some $\alpha^{\prime}, \beta^{\prime} \in \Pi_{W_{1}}$, by the admissibility of $c_{1}$. Now we need to "pull back" (4) from $\Pi_{W_{1}}$ to $\Pi_{W}$, and for this it suffices to prove that $\gamma \sim \delta \Longrightarrow \iota_{1}(\gamma) \sim \iota_{1}(\delta)$ and $\gamma \rightsquigarrow_{1} \delta \Longrightarrow \iota_{1}(\gamma) \rightsquigarrow_{1} \iota_{1}(\delta)$ for all $\gamma, \delta \in \Pi_{W_{1}}$. The intuitive reason behind both implications is the same: the interval $\left[\iota_{1}(\gamma), \top\right] \subseteq \Pi_{W}$ differs from $\left[\gamma, \top_{1}\right] \subseteq \Pi_{W_{1}}$ only in some monochromatic part around the top (all colors are $\varnothing$ there), and similarly for $\delta$. We shall check the details formally below.

First assume that $\gamma \sim \delta$, i.e., there exists a color-preserving isomorphism $\varphi:\left[\gamma, \top_{1}\right] \rightarrow$ $\left[\delta, \top_{1}\right]$. Since $\Pi_{|\gamma|} \cong\left[\gamma, \top_{1}\right] \cong\left[\delta, \top_{1}\right] \cong \Pi_{|\delta|}$, we must have $|\gamma|=|\delta|$; moreover, by Ore's theorem (Theorem 2), $\varphi=\widetilde{\pi}$ for some bijection $\pi: \gamma \rightarrow \delta$. Let us extend $\pi$ to a bijection $\tau: \iota_{1}(\gamma) \rightarrow \iota_{1}(\delta)$ as follows:

$$
\tau(U)= \begin{cases}\pi(U), & \text { if } \left.U \in \gamma \text { (i.e., } U \subseteq W_{1}\right) \\ U, & \text { otherwise (i.e., if } \left.U=\{w\} \text { for some } w \in W_{2}\right) .\end{cases}
$$

We claim that $\widetilde{\tau}$ is a color-preserving isomorphism between the intervals $\left[\iota_{1}(\gamma), \top\right]$ and $\left[\iota_{1}(\delta), \top\right]$. Indeed, if $\iota_{1}(\gamma) \leq \xi \leq \omega_{1}$, then $\widetilde{\tau}(\xi)=\iota_{1}(\widetilde{\pi}(\eta))$, where $\eta=\iota_{1}^{-1}(\xi)$, and thus $c(\widetilde{\tau}(\xi))=c_{1}(\widetilde{\pi}(\eta))=c_{1}(\eta)=c(\xi)$, by the definition of $c$, and by the color-preserving property of $\widetilde{\pi}$. Now let $\xi \in\left[\iota_{1}(\gamma), \top\right]$ but $\xi \not \leq \omega_{1}$. Then $\tilde{\tau}(\xi) \not \omega_{1}$, hence $c(\widetilde{\tau}(\xi))=\varnothing=c(\xi)$. Therefore, $\widetilde{\tau}$ indeed preserves colors, and this proves that $\iota_{1}(\gamma) \sim \iota_{1}(\delta)$.

Next assume that $\gamma \rightsquigarrow_{1} \delta$, i.e., there exists $\vartheta \in \Pi_{W_{1}}$ such that $\gamma \leq \vartheta \prec \top_{1}$, $\gamma \prec \delta \not \leq \vartheta$, and $c_{1}(\eta)=c_{1}(\eta \wedge \vartheta)$ for all $\eta \in\left[\gamma, \top_{1}\right]$. Suppose (without loss of generality) that $\gamma=\left\{V_{1}, \ldots, V_{k}\right\}, \vartheta=\left\{V_{1}, V_{2} \cup \cdots \cup V_{k}\right\}=\left\{V_{1}, W_{1} \backslash V_{1}\right\}$, and let $\Theta=\left\{V_{1}, W \backslash V_{1}\right\}$. Clearly, we have $\iota_{1}(\gamma) \leq \Theta \prec \top$ and $\iota_{1}(\gamma) \prec \iota_{1}(\delta) \not \leq \Theta$. We need to verify that $c(\xi \wedge \Theta)=c(\xi)$ for every $\xi \in\left[\iota_{1}(\gamma), \top\right]$. If $\iota_{1}(\gamma) \leq \xi \leq \omega_{1}$, then $c(\xi \wedge \Theta)=c\left(\xi \wedge \omega_{1} \wedge \Theta\right)=c\left(\xi \wedge \iota_{1}(\vartheta)\right)$, since $\iota_{1}(\vartheta)=\omega_{1} \wedge \Theta$. Setting $\eta=\iota_{1}^{-1}(\xi)$, we can conclude

$$
c(\xi \wedge \Theta)=c\left(\iota_{1}(\eta) \wedge \iota_{1}(\vartheta)\right)=c\left(\iota_{1}(\eta \wedge \vartheta)\right)=c_{1}(\eta \wedge \vartheta)=c_{1}(\eta)=c(\xi) .
$$

If $\xi \in\left[\iota_{1}(\gamma)\right.$, $\left.\top\right]$ but $\xi \not \leq \omega_{1}$, then $\xi \wedge \Theta \not \leq \omega_{1}$, hence $c(\xi \wedge \Theta)=\varnothing=c(\xi)$. Therefore, $\iota_{1}(\gamma) \rightsquigarrow_{1} \iota_{1}(\delta)$, as claimed. 


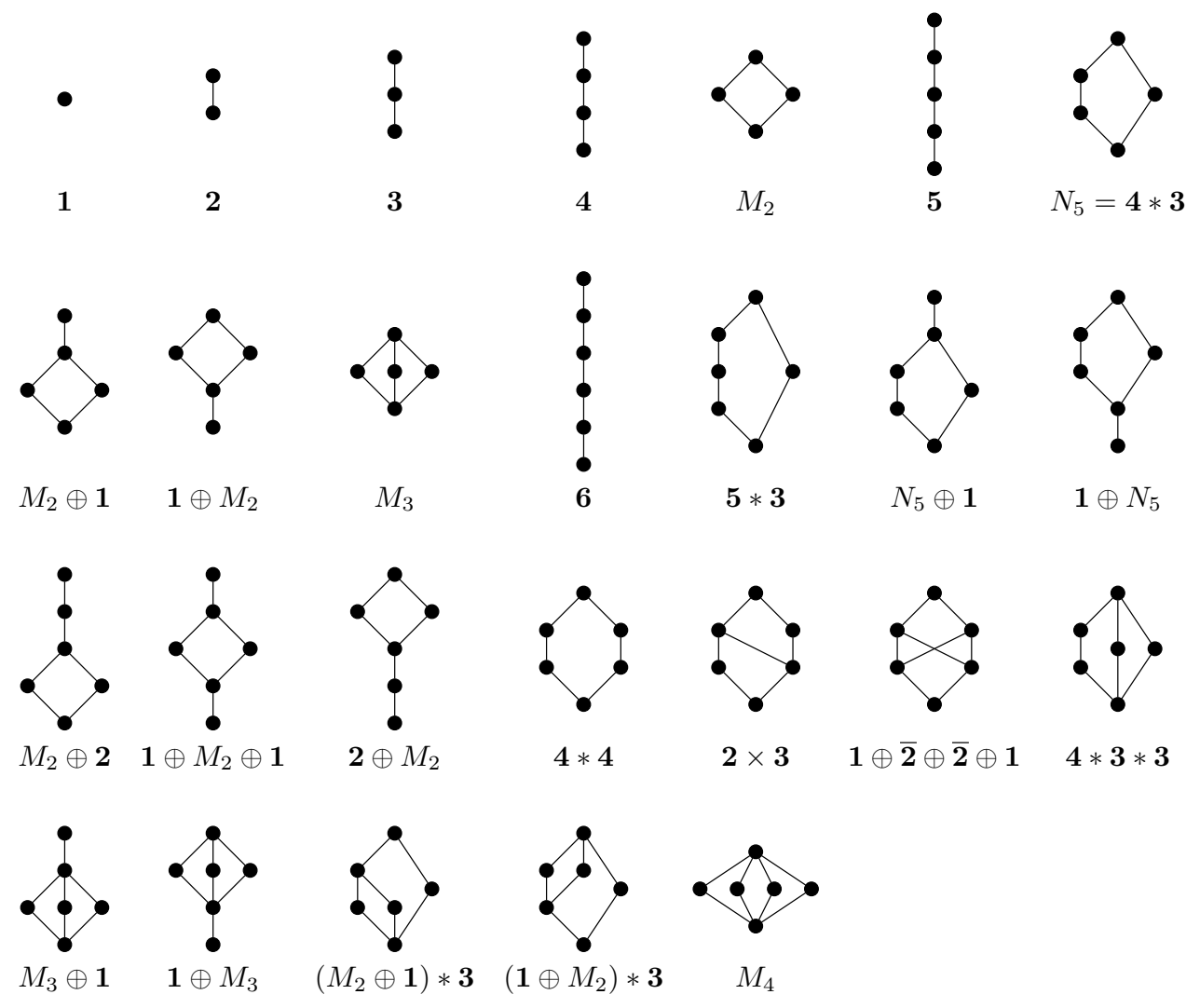

Figure 5. Bounded posets on up to six points.

Starting from the examples of Theorem 19, one can build many minor posets using the constructions of Theorems 23 and 25. For example, the poset of Figure 1 can be constructed as $\mathbf{3} *\left(M_{2} \oplus \mathbf{1}\right)$. In our last theorem we prove that all bounded posets on up to 6 elements are minor posets.

Theorem 26. All bounded posets with at most 6 elements are minor posets.

Proof. We have enumerated the bounded posets on up to six points in Figure 5. The list is exhaustive; the reader may compare the list with the numbers given by Brinkmann and McKay 3. As can be seen from Figure 5, the posets with at most 6 elements can be built using the constructions of Theorems 19, 23 and 25, with the exception of the following: $\mathbf{2} \times \mathbf{3}, \mathbf{1} \oplus \overline{\mathbf{2}} \oplus \overline{\mathbf{2}} \oplus \mathbf{1}$ and $\mathbf{1} \oplus N_{5}$. Therefore, in order to prove the result, we must provide, for each one of these three exceptional posets, an admissible coloring $c$ of a partition lattice $\Pi_{n}$ such that the quotient $\Pi_{n} / \operatorname{ker} c$ is dually isomorphic to the desired poset.

For $\mathbf{2} \times \mathbf{3}$, we color $\Pi_{4}$ as follows (see Figure 9 ). The bottom element of $\Pi_{4}$ is white. The partitions $1 / 23 / 4$ and $14 / 2 / 3$ are yellow; the other partitions with three blocks are green. The partition 14/23 is blue; the other partitions with two blocks are red. The top element is black. Then $\Pi_{4} / \operatorname{ker} c$ is (dually) isomorphic to $\mathbf{2} \times \mathbf{3}$.

For $\mathbf{1} \oplus \overline{\mathbf{2}} \oplus \overline{\mathbf{2}} \oplus \mathbf{1}$, we color $\Pi_{4}$ as follows (see Figure 10 ):

$$
\begin{aligned}
1 / 2 / 3 / 4 & \mapsto \text { white, } \\
1 / 23 / 4,14 / 2 / 3,1 / 24 / 3 & \mapsto \text { yellow, } \\
13 / 2 / 4,12 / 3 / 4,1 / 2 / 34 & \mapsto \text { green, } \\
14 / 23,13 / 24,12 / 34 & \mapsto \text { blue, } \\
1 / 234,134 / 2,124 / 3,123 / 4 & \mapsto \text { red, } \\
1234 & \mapsto \text { black. }
\end{aligned}
$$


Then $\Pi_{4} / \operatorname{ker} c$ is (dually) isomorphic to $\mathbf{1} \oplus \overline{\mathbf{2}} \oplus \overline{\mathbf{2}} \oplus \mathbf{1}$.

For $1 \oplus N_{5}$, we color $\Pi_{5}$ according to the number and sizes of blocks and the size of the block containing 5 as follows:

$$
\begin{aligned}
\bullet / \bullet / \bullet / \bullet / 5 & \mapsto \text { white, } \\
\bullet \bullet / \bullet / \bullet / 5 & \mapsto \text { yellow }, \\
\bullet / \bullet / \bullet / \bullet 5 & \mapsto \text { green, } \\
\bullet \bullet \bullet / \bullet / 5 & \mapsto \text { blue, } \\
\bullet / \bullet / \bullet \bullet 5, \bullet / \bullet \bullet / \bullet 5, \bullet / \bullet \bullet 5, \bullet \bullet \bullet / \bullet 5 & \mapsto \text { red } \\
\bullet \bullet / \bullet \bullet / 5, \bullet \bullet \bullet \bullet / 5, \bullet \bullet / \bullet \bullet 5, \bullet \bullet \bullet 5 & \mapsto \text { black }
\end{aligned}
$$

Figure 11 shows a schematic diagram of $\Pi_{5}$ with this coloring. Each edge of the diagram has a label indicating the number of upper covers of a partition belonging to a given group. For example, if $\alpha$ is a partition of type $\bullet / \bullet / \bullet \bullet 5$, then $\alpha$ has 2 upper covers of type $\bullet / \bullet \bullet \bullet 5$ and 1 upper cover of type $\bullet \bullet / \bullet \bullet 5$. This information is sufficient to verify that the coloring is admissible, and it is clear that $\Pi_{5} / \operatorname{ker} c$ is isomorphic to $N_{5} \oplus \mathbf{1}$, hence dually isomorphic to $\mathbf{1} \oplus N_{5}$.

\section{Conclusion}

We presented an abstract characterization of minor posets in terms of admissible colorings of partition lattices, and we provided many examples of minor posets. These results suggest some natural questions worth further investigations:

- Can we add a new bottom element to a minor poset, i.e., is it true that $P \in \mathcal{M}$ implies $\mathbf{1} \oplus P \in \mathcal{M}$ ? In particular, is $\mathbf{2} \oplus N_{5}$ a minor poset?

- Is the set of minor posets closed under direct products? In particular, is $\mathbf{3} \times \mathbf{3}$ a minor poset?

- For a given natural number $n$, what is the smallest $k$ such that every minor poset of size $n$ can be realized by a coloring of $\Pi_{k}$ ?

- Is it decidable whether a given finite bounded poset is a minor poset?

- After all, is every finite bounded poset a minor poset?

\section{ACKNOWLEDGEMENTS}

Research supported by the Hungarian National Research, Development and Innovation Office (NKFIH grants no. K104251 and K115518) and by the János Bolyai Research Scholarship. This work was developed during the authors' mutual visits to the Technische Universität Dresden and the University of Szeged. The authors would like to thank the anonymous reviewers for their valuable comments and suggestions for improving the paper. 

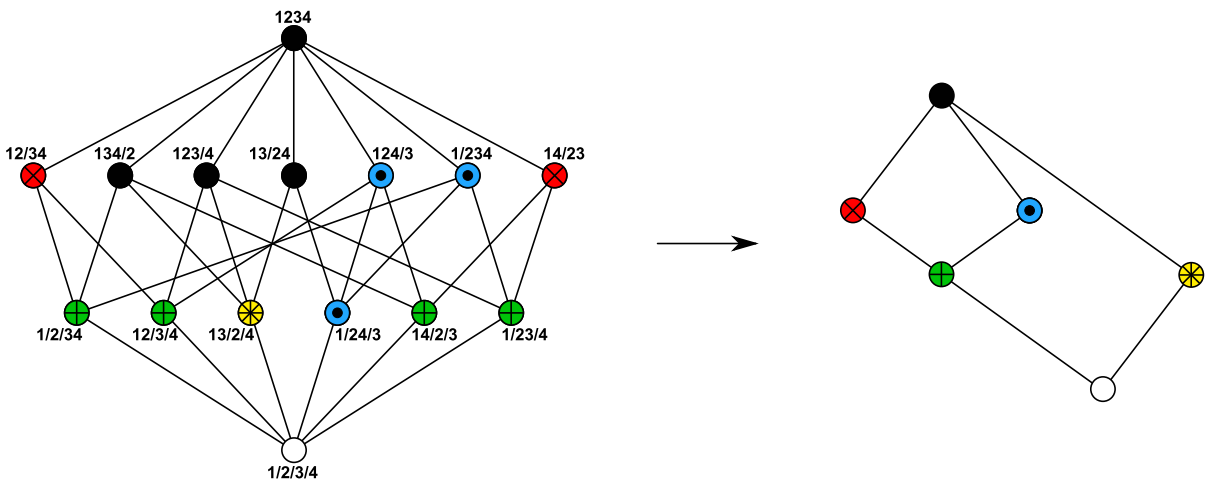

FiguRE 6 . The coloring of $\Pi_{4}$ that corresponds to Figure 1 .
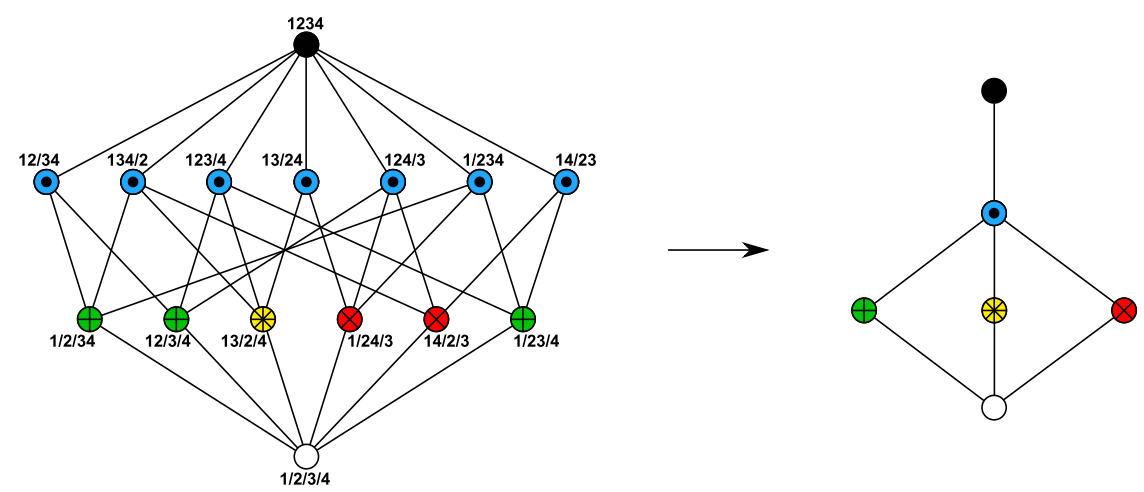

Figure 7. A coloring of $\Pi_{4}$ with quotient $M_{3} \oplus \mathbf{1}$.
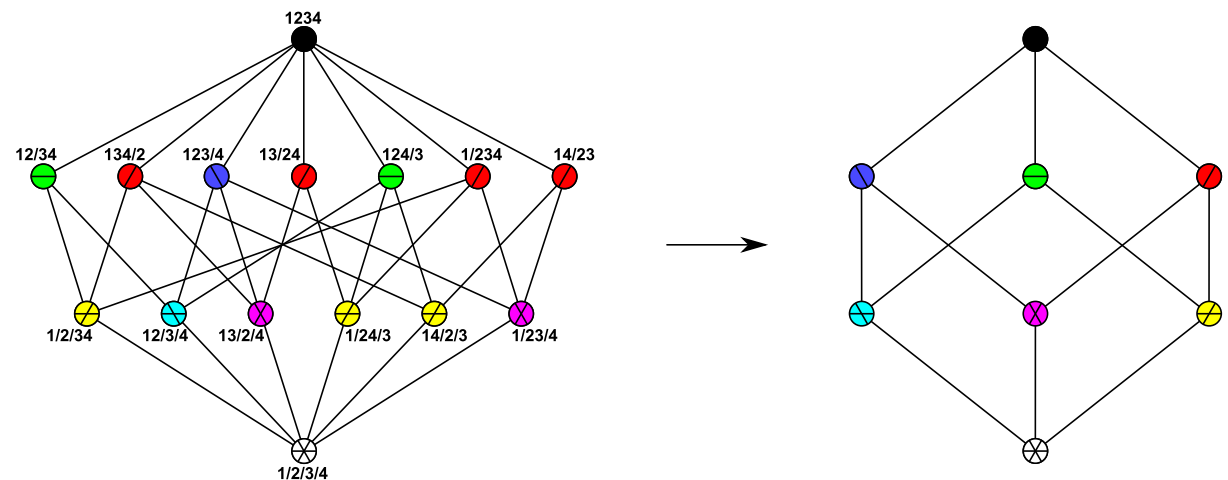

Figure 8. A coloring of $\Pi_{4}$ with quotient $\mathbf{2}^{3}$. 


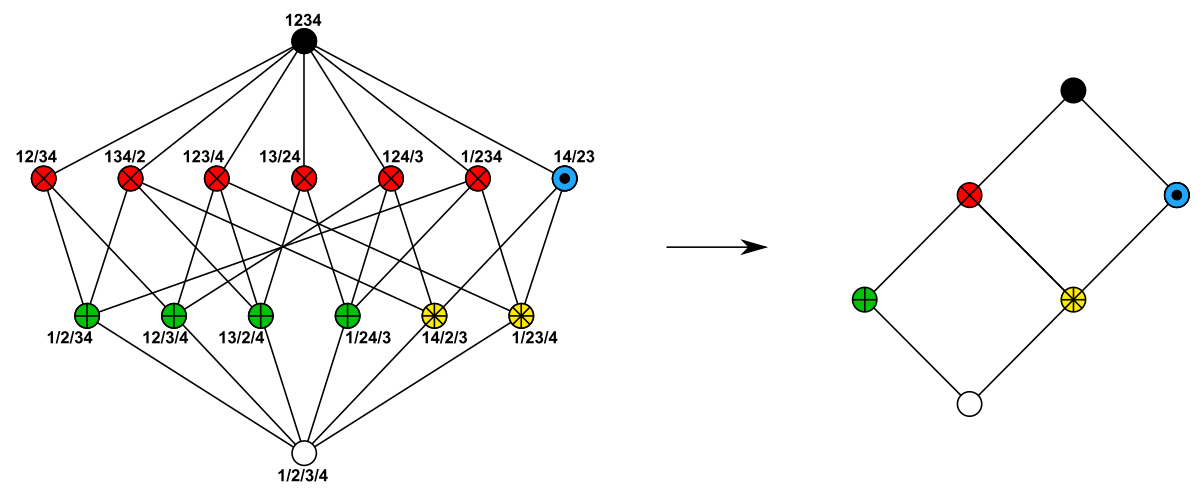

FiguRE 9 . A coloring of $\Pi_{4}$ with quotient $\mathbf{2} \times \mathbf{3}$.
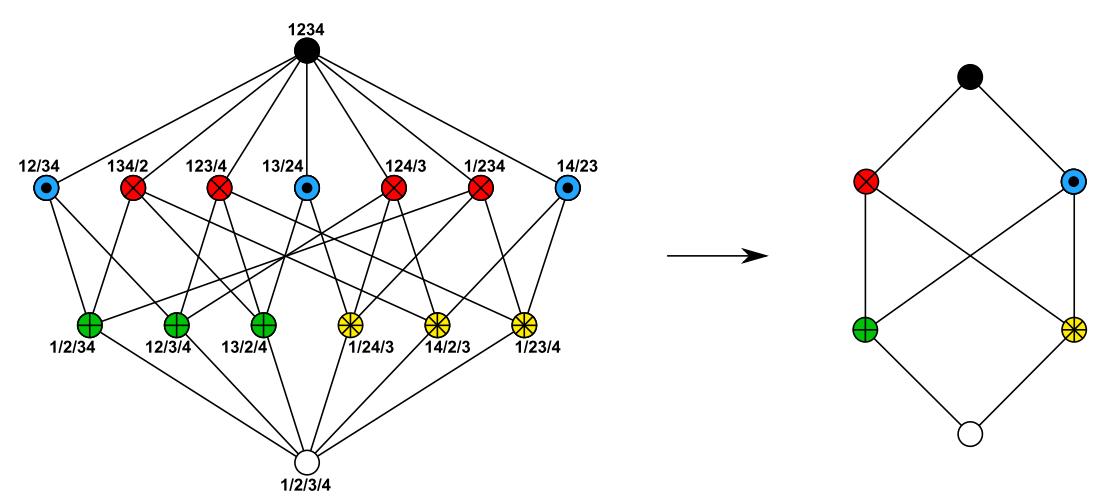

Figure 10. A coloring of $\Pi_{4}$ with quotient $\mathbf{1} \oplus \overline{\mathbf{2}} \oplus \overline{\mathbf{2}} \oplus \mathbf{1}$.
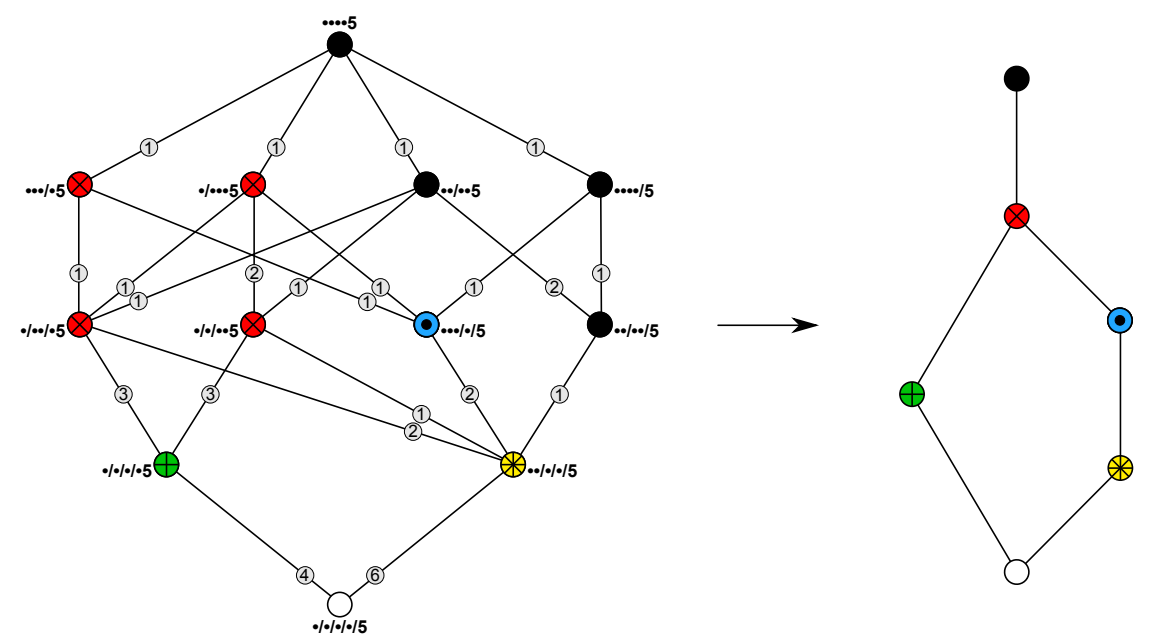

Figure 11. A coloring of $\Pi_{5}$ with quotient $N_{5} \oplus \mathbf{1}$. 


\section{REFERENCES}

[1] Beran, L., Ježek, J.: On embedding of lattices in simple lattices. Acta Univ. Carolinae-Math. et Phys. 13, 87-89 (1972)

[2] Birkhoff, G.: Lattice Theory. American Mathematical Society Colloquium Publications, vol. 25. American Mathematical Society, Providence RI (1967)

[3] Brinkmann, G., McKay, B.D.: Posets on up to 16 points. Order 19, 147-179 (2002)

[4] Couceiro, M., Lehtonen, E., Waldhauser, T.: Parametrized arity gap. Order 30, 557-572 (2013)

[5] Couceiro, M., Pouzet, M.: On a quasi-ordering on Boolean functions. Theoret. Comput. Sci. 396, 71-87 (2008)

[6] Lehtonen, E., Szendrei, Á.: Partial orders induced by quasilinear clones. Proceedings of the Salzburg Conference 2011 (AAA81), Contributions to General Algebra vol. 20, pp. 51-84. Verlag Johannes Heyn, Klagenfurt (2012)

[7] Lehtonen, E., Waldhauser, T.: Posets of minors of functions in multiple-valued logic. Proceedings of the 47th IEEE International Symposium on Multiple-Valued Logic (ISMVL 2017), pp. 43-48. IEEE Computer Society (2017)

[8] Ore, O.: Theory of equivalence relations. Duke Math. J. 9, 573-627 (1942)

[9] Post, E. L.: The two-valued iterative systems of mathematical logic. Annals of Mathematics Studies, no. 5, Princeton University Press, Princeton, N. J. (1941)

[10] Willard, R.: Essential arities of term operations in finite algebras. Discrete Math. 149, 239-259 (1996)

(E. Lehtonen) Institut für Algebra, Technische Universität Dresden, 01062 Dresden, GERMANY

E-mail address: Erkko.Lehtonen@tu-dresden.de

(T. Waldhauser) Bolyai Institute, University of Szeged, Aradi vértanúk tere 1, H-6720 SzEGED, Hungary

E-mail address: twaldha@math.u-szeged.hu 